\title{
Correction to: Cancer: The Enemy from Within
}

\section{Correction to: C. Compton, Cancer: The Enemy from Within, https://doi.org/10.1007/978-3-030-40651-6}

The original version of the book, unfortunately, contained errors. The version supplied here has been corrected and approved by the author.

The errors in the scientific content are listed below.

Page 14, In chapter 1 "For unknown reasons, the only malignant neoplasms that do metastasize are those of the central nervous system and the class of skin cancers known as basal cell carcinomas."

The chapter has now been corrected, and the updated sentence should read as follows:

"For unknown reasons, the only malignant neoplasms that do not metastasize are those of the central nervous system and the class of skin cancers known as basal cell carcinomas."

Page 31, Chapter 2, Fig 2.5: Under germline mutations on the right-hand side at the top, the second bullet has been changed from "Cannot herited" to "Heritable." The correct presentation is provided here.

The updated version of these chapter can be found at https://doi.org/10.1007/978-3-030-40651-6_1 https://doi.org/10.1007/978-3-030-40651-6_2 https://doi.org/10.1007/978-3-030-40651-6_3 https://doi.org/10.1007/978-3-030-40651-6_6 https://doi.org/10.1007/978-3-030-40651-6 
Somatic mutations

- Occur in nongermline tissues

- Cannot be inherited

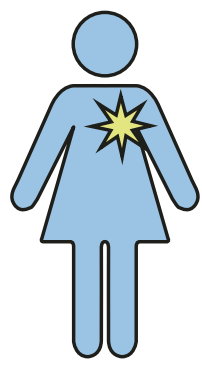

Mutation in tumor only (for example, breast)
Germline mutations

- Present in egg or spem

- Heritable

- Cause cancer family syndrome
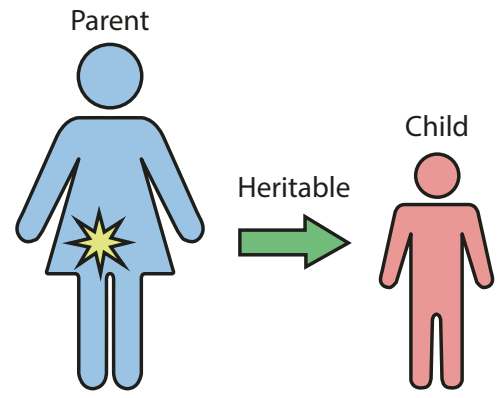

Mutation in

All cells

egg or sperm affected in offspring

Page 78: In the sentence "The upregulation of glucose..." the term "c-myc oncogene" has been changed to "c-myc proto-oncogene".

Page 78: In the sentence "Normal aerobic metabolism..." the term "32 ATP" has been corrected to "36 ATP". "The same was fixed in Figure 3.25".

Page 139, Chapter 6: The sentence "Neither of these errors is unacceptable" has been changed to "Neither of these errors is acceptable". 